\title{
Stress Induces Release of Extracellular Vesicles by Trypanosoma cruzi Trypomastigotes
}

\author{
Camilla Ioshida Vasconcelos, ${ }^{1}$ A Cronemberger-Andrade, ${ }^{2}$ Normanda Souza-Melo, ${ }^{3}$ \\ Juliana Terzi Maricato, ${ }^{4}$ Patrícia Xander, ${ }^{1}$ Wagner Luiz Batista, ${ }^{1}$ Rodrigo Pedro Soares, ${ }^{5}$ \\ Sergio Schenkman $\mathbb{D}^{3},{ }^{3}$ and Ana Claudia Torrecilhas $\mathbb{D}^{1}$ \\ ${ }^{1}$ Departamento de Ciências Farmacêuticas, UNIFESP, Rua São Nicolau, 210, 09913-030, Diadema, São Paulo, Brazil \\ ${ }^{2}$ Cell Therapy Institute, Spinal Cord Injury and Tissue Regeneration Center Salzburg (SCI-TReCS), Paracelsus Medical \\ University (PMU), 5020 Salzburg, Austria \\ ${ }^{3}$ Departamento de Microbiologia, Imunologia e Parasitologia, UNIFESP, Rua Pedro de Toledo, 669, 04039-032 São Paulo, Brazil \\ ${ }^{4}$ Departamento de Microbiologia, Imunologia e Parasitologia, UNIFESP, Rua Botucatu, 862, 04023-062 São Paulo, Brazil \\ ${ }^{5}$ Instituto René Rachou/FIOCRUZ-MG, Av. Augusto de Lima, 1715, 30190-009 Belo Horizonte, Minas Gerais, Brazil
}

Correspondence should be addressed to Sergio Schenkman; sschenkman@unifesp.br

and Ana Claudia Torrecilhas; ana.torrecilhas@unifesp.br

Received 14 June 2021; Accepted 26 August 2021; Published 24 September 2021

Academic Editor: Ilaria Roato

Copyright (C) 2021 Camilla Ioshida Vasconcelos et al. This is an open access article distributed under the Creative Commons Attribution License, which permits unrestricted use, distribution, and reproduction in any medium, provided the original work is properly cited.

\begin{abstract}
All extracellular forms of Trypanosoma cruzi, the causative agent of Chagas disease, release extracellular vesicles (EVs) containing major surface molecules of the parasite. EV release depends on several mechanisms (internal and external). However, most of the environmental conditions affecting this phenomenon are still unknown. In this work, we evaluated EV release under different stress conditions and their ability to be internalized by the parasites. In addition, we investigated whether the release conditions would affect their immunomodulatory properties in preactivated bone marrow-derived macrophages (BMDM). Sodium azide and methyl-cyclo- $\beta$-dextrin $(\mathrm{CDB})$ reduced EV release, indicating that this phenomenon relies on membrane organization. EV release was increased at low temperatures $\left(4^{\circ} \mathrm{C}\right)$ and acidic conditions $(\mathrm{pH} 5.0)$. Under this $\mathrm{pH}$, trypomastigotes differentiated into amastigotes. EVs are rapidly liberated and reabsorbed by the trypomastigotes in a concentration-dependent manner. Nitrosative stress caused by sodium nitrite in acid medium or $S$-nitrosoglutathione also stimulated the secretion of EVs. EVs released under all stress conditions also maintained their proinflammatory activity and increased the expression of iNOS, Arg 1, IL-12, and IL-23 genes in IFN- $\gamma$ and LPS preactivated BMDM. In conclusion, our results suggest a budding mechanism of release, dependent on the membrane structure and parasite integrity. Stress conditions did not affect functional properties of EVs during interaction with host cells. EV release variations under stress conditions may be a physiological response against environmental changes.
\end{abstract}

\section{Introduction}

The flagellated protozoan Trypanosoma cruzi is the etiological agent of Chagas disease, affecting 8 million people worldwide. Approximately 100 million people are at risk of infection, causing about 2,000 deaths per year. These circumstances make this disease a serious health problem $[1,2]$. In the bloodstream of the mammalian vertebrate host, trypomastigote forms invade various cell types, differentiate into amastigotes, and proliferate in their cytoplasm.

Trypomastigotes derived from infected mammalian cell cultures release extracellular vesicles (EVs) in the culture medium. They express major protozoan surface molecules [2], including mucin-like glycoproteins, glycosylphosphatidyl inositol phospholipids (GIPLs), and members of the gp85/trans-sialidase (TS) superfamily of glycoproteins [3]. 
EV release promotes parasite infectivity and modulates the host's innate and acquired immune responses [4-10]. However, mechanisms involved in EV release by the parasites facing adverse environmental conditions are still poorly understood. Those may include diverse body fluids (e.g., blood), extracellular matrix, and contact with chemical agents. As a part of a wider study on T. cruzi EVs, we sought to characterize their release under different conditions (temperature, $\mathrm{pH}$, and chemical agents). We also evaluated if $T$. cruzi EVs released under stress conditions were functionally affected during interaction with bone marrow-derived macrophages.

\section{Material and Methods}

2.1. Animal Ethics. The experimental procedures used in this study were approved by the Animal Use Ethics Committee (CEUA) of the Federal University of São Paulo (http:// www.unifesp.br/reitoria/ceua) under protocol \# 9254110216.

2.2. Parasite Cultures. Trypomastigotes (Y-strain) were obtained from the supernatant of infected LLC-MK ${ }_{2}$ cells maintained in low-glucose DMEM (Vitrocell Embriolife), supplemented with $10 \%$ fetal bovine serum (SFB) (Vitrocell Embriolife). Trypomastigotes were collected from the cell culture medium from the fifth to the ninth day after infection by centrifugation $(1,000 \times g$ for $15 \mathrm{~min})$. They were washed with PBS, and the pellet containing the parasites was resuspended in $1 \mathrm{~mL}$ DMEM. Parasite concentration was estimated using a Neubauer (Reichert) chamber.

2.3. EV Release Assays. Trypomastigotes $\left(1 \times 10^{7}\right)$ were incubated for $2 \mathrm{~h}$ in DMEM with/without FBS containing 5\% glucose, at distinct temperatures and $\mathrm{pH}$ levels, and/or in the presence of metabolic inhibitors and nitroxidative compounds $\left(\mathrm{NaN}_{3}\right.$ and $\left.\mathrm{NaNO}_{2}\right)$ and methyl- $\beta$ cyclodextrin (CBD). The assays were always performed in triplicate. After incubation, trypomastigotes were centrifuged $(1,000 \times g$ for $15 \mathrm{~min})$ (MiniSpin plus, Eppendorf) and supernatants were collected for measuring EVs' size and concentration. To determine viability, $90 \mu \mathrm{L}$ of the parasite's suspension was added to each well of a 96-well plate and mixed with $10 \mu \mathrm{L}$ of the PrestoBlue (Thermo Fisher Scientific). Plates were incubated $\left(37^{\circ} \mathrm{C}, 2 \mathrm{~h}\right)$, and fluorescence was detected by excitation at $560 \mathrm{~nm}$ and emission at $590 \mathrm{~nm}$ (Synergy HT, BioTek). Parasites incubated without any agent were used as negative controls, and the results were expressed as relative fluorescence units. In parallel, trypomastigotes were centrifuged $(3,000 \times g, 5 \mathrm{~min})$ and the supernatant was discarded. The pellet was resuspended in PBS, dried on glass coverslips, fixed in methanol, and washed with $2 \mathrm{X}$ running water. Then, parasites were stained with Giemsa (Merck) diluted 1:10 in running water and mounted with Entellan (Merck). Images were acquired under an optical microscope. 300 parasites/slide were counted, and T. cruzi morphology was analyzed (Imager.A2, Zeiss).

2.4. Isolation of T. cruzi Trypomastigote EVs. The total shed material released by the trypomastigotes was centrifuged $(10,000 \times g, 15 \mathrm{~min})$. After centrifugation, the supernatant was filtered using a $0.22 \mu \mathrm{m}$ filter (Sarstedt) and ultracentrifuged $\left(100,000 \times g, 1 \mathrm{~h}, 4^{\circ} \mathrm{C}\right.$ ) (Sorvall WX Ultra Series 80, rotor T890, Thermo Scientific). The pellet containing EVs was resuspended in $5 \mathrm{~mL}$ of sterile PBS and submitted to a new ultracentrifugation under the same conditions. EVs were recovered from the pellet, resuspended in sterile PBS, and stored at $4^{\circ} \mathrm{C}$. The procedures follow the Minimal Information for Studies of Extracellular Vesicles 2018 [11].

2.5. Scanning Electronic Microscopy (SEM). Poly L-lysine solution $(200 \mu \mathrm{L})$ at $0.01 \%$ (Sigma-Aldrich), prefiltered through a $0.22 \mu \mathrm{m}$ pore size filter, was added onto circular coverslips ( $13 \mathrm{~mm}$, Glasscyto). After $30 \mathrm{~min}$, the poly Llysine solution was removed, and the wells washed with filtered water and covered with $50 \mu \mathrm{L}$ containing $1 \times 10^{6}$ trypomastigotes in PBS. After 30 min incubation at room temperature (RT), parasites were fixed $(2.5 \%$ glutaraldehyde in $0.1 \mathrm{M}$ sodium cacodylate buffer), followed by $1 \mathrm{~h}$ incubation (RT). Samples were stored $\left(4^{\circ} \mathrm{C}\right)$ prior to electron microscopy scanning at the Federal University of São Paulo facility (CEME, UNIFESP), as previously described [5].

2.6. Nanoparticle Tracking Analysis (NTA). The material released by the parasites was characterized by size and concentration using NTA (NanoSight, NS 300, Malvern, equipped with a CCD camera). Samples were either analyzed pure or diluted $(1: 10)$ in filtered PBS. Each capture was performed in triplicate always using the same threshold $\left(24.7-24.9^{\circ} \mathrm{C}, 30 \mathrm{sec}\right.$, camera level set to 10$)$. Results were expressed in concentration (particles/mL), size, and distribution of EVs using NTA software (version 2.3 build 0017).

2.7. EV Labeling and Reincorporation in the Parasite. Purified EVs were concentrated by ultracentrifugation $(18 \mathrm{~h}$ at $100,000 \times g)$ and particles $\left(5 \times 10^{8} / \mathrm{mL}\right.$ incubated with $2 \mu \mathrm{M}$ PKH26 Red Fluorescent cell dye (MINI26, SigmaAldrich). After $30 \mathrm{~min}$ at $37^{\circ} \mathrm{C}$ (dark), the mixture was diluted $5 \mathrm{x}$ with PBS and ultracentrifuged $(18 \mathrm{~h}, 100,000 \times \mathrm{g})$. The pellet was resuspended with Diluent $C$, and the number of EVs determined by NTA. Different concentrations of labeled EVs were incubated with trypomastigotes $\left(1 \times 10^{6}\right.$ $/ \mathrm{mL}$ ) in DMEM containing 5\% glucose. Parasites were collected by centrifugation at different time points $(5 \mathrm{~min}$ at $1,000 \times g$ ), washed once with PBS, and resuspended in PBS$0.5 \%$ p-formaldehyde. Samples were analyzed by flow cytometry (Fortessa, BD).

2.8. Bone Marrow-Derived Macrophage (BMDM) Interaction with T. cruzi EVs. Bone marrow cells obtained from 6- to 8week-old female $\mathrm{C} 57 \mathrm{Bl} / 6$ mice were submitted to differentiation in macrophages by culturing for 7 days in RPMI (Vitrocell Embriolife) culture medium containing 10\% FBS, with the addition of L929 supernatant as previously described [12]. $1 \times 10^{6}$ macrophages were plated in 6-well plates. The cells were stimulated for $12 \mathrm{~h}$ with $50 \mathrm{ng} / \mathrm{mL}$ IFN- $\gamma$ and $500 \mathrm{ng} / \mathrm{mL}$ LPS (L2330, Sigma-Aldrich). These preactivated macrophages were then incubated for $24 \mathrm{~h}$ with $1 \times 10^{8}$ EVs released by $T$. cruzi from different conditions. 
TABLE 1: Oligonucleotides used for amplification, sequence, and gene of each target gene.

\begin{tabular}{lcc}
\hline Oligonucleotide & Sequence & Gene \\
\hline Arg1Fow & $5^{\prime}$-GAGACAGGGAAGTCTGAAGCAC & Arginase 1 \\
Arg1Rev & $5^{\prime}$-CATTGGCTTGCGAGACGTAGAC & Arginase 1 \\
iNOS Fow & $5^{\prime}$-ATGGACCAGTATAAGGCAAGC & iNOS \\
iNOS Rev & $5^{\prime}$-GCTCTGGATGAGCCTATATTG & iNOS \\
IL-23p19 Fow & $5^{\prime}$ AATAATGTGCCCCGTATCCAG & IL-23p19 \\
IL-23p19Rev & $5^{\prime}$ GCTCCCCTTTGAAGATGTCAG & IL-23p19 \\
IL-12p35 Rev & $5^{\prime}$-ACGAGAGTTGCCTGGCTACTA & IL-12p35 \\
IL-12p35Fow & $5^{\prime}$-CCTCATAGATGCTACCAAGGCAC & IL-12p35 \\
IL-12p40 Fow & $5^{\prime}$-TTGAACTGGCGTTGGAAGCACG & IL-12p40 \\
IL-12p40 Rev & $5^{\prime}$-CCACCTGTGAGTTCTTCAAAGGC & IL-12p40 \\
Ym2Fow & $5^{\prime}$-GTGACCCTACTGTTAGTGCTGG & YM2 \\
Ym2Rev & $5^{\prime}$ CGGGAAGACAATAACTGCACCC & YM2 \\
IL-10 Fow & $5^{\prime}$ GACTTTAAGGGTTACCTGGGTTG & IL-10 \\
IL-10 Rev & $5^{\prime}$ TCACATGCGCCTTGATGTCTG & IL-10 \\
GAPDH forward & $5^{\prime}$ AAATGGTGAAGGTCGGTGTG & GAPDH \\
GAPDH reverse & $5^{\prime}$ TGAGGGGTCGTTGATGG & GAPDH \\
\hline
\end{tabular}

The cell culture supernatant was stored at $4^{\circ} \mathrm{C}$ for analysis by NTA, and the cells were extracted for cytokine analysis.

2.9. Gene Expression by $q R T-P C R$. RNA was extracted from BMDM using RNeasy Plus Microkit (QIAGEN). All samples were also submitted to fluorometry analysis and DNAse treatment. Complementary DNA (cDNA) synthesis was performed using SuperScript II reverse transcriptase kit (Life Technologies), as previously described [13, 14]. Gene expression (qRT-PCR) used SYBR Green-based system detection (Applied Biosystems, Life Technologies). Each reaction was composed of $2 \mu \mathrm{M}$ of forward and reverse oligonucleotides for each target gene (expression levels were normalized to those of the GAPDH control), $10 \mu \mathrm{L}$ of the SYBR Green PCR master mix (Applied Biosystems), and $3 \mu \mathrm{L}$ of cDNA $1: 2.5$ diluted cDNA. Cycling reactions were carried out in the Applied Biosystems 7500 System (Applied Biosystems) starting with one cycle of $50^{\circ} \mathrm{C}(2 \mathrm{~min})$ and $95^{\circ} \mathrm{C}$ $(1 \mathrm{~min})$, followed by 45 cycles at $95^{\circ} \mathrm{C}(15 \mathrm{sec})$ and $60^{\circ} \mathrm{C}$ (1 min). Melting curves were determined with an additional cycle of $95^{\circ} \mathrm{C}(15 \mathrm{sec}), 60^{\circ} \mathrm{C}(20 \mathrm{sec})$, and $95^{\circ} \mathrm{C}(15 \mathrm{sec})$, as previously described [13]. The reference genes using the $2^{(-\Delta \Delta \mathrm{Ct})}$ cycle threshold method, as previously described $[13,15,16]$. All qRT-PCR procedures were performed following the MIQE guidelines [16]. Each reaction was made in triplicate. The oligonucleotides used for amplification of each target gene are described in Table 1.

2.10. Statistical Analysis. Statistical analysis was performed using GraphPad Prism version 6 (GraphPad Software, La Jolla, CA, United States). Data were analyzed using one-way ANOVA test. $p$ values $<0.05$ were considered significant.

\section{Results}

3.1. Kinetics of EV Release under Different Conditions. To follow the process of EV release by trypomastigotes derived from infected mammalian cells, their kinetics under different conditions were evaluated. EVs were collected after 30 minutes of parasite resuspension in the serum-free DMEM containing $5 \%$ glucose. The release was higher at $4^{\circ} \mathrm{C}$ (Figure 1(a)). At $37^{\circ} \mathrm{C}$, EVs increased progressively in the supernatant and reached a maximal value at 120 minutes. The number of particles released by the parasites was significantly higher after incubation of trypomastigotes at $4^{\circ} \mathrm{C}$ compared to $26^{\circ} \mathrm{C}$ and $37^{\circ} \mathrm{C}$ (Figure 1(b)), without changes in size (Figure 1(c)). In all cases, the size of EVs was approximately $150 \mathrm{~nm}$, as detected by NTA. SEM also showed a larger amount of material associated with the parasites at $4^{\circ} \mathrm{C}$ compared to those at $26^{\circ} \mathrm{C}$ and $37^{\circ} \mathrm{C}$ (Figures $1(\mathrm{~d})-1(\mathrm{f})$ ). No significant differences in the PrestoBlue signal activity, suggesting that the cell metabolic activity was not largely affected in trypomastigotes (Figure $1(\mathrm{~g})$ ) that were preincubated for $2 \mathrm{~h}$ from 4 to $37^{\circ} \mathrm{C}$ as shown underneath each image.

To evaluate parasites' ability to release/uptake EVs, they were labeled with PKH26 fluorescent probe, a dye that is incorporated into the glycocalyx of the membranes. Labeled EVs were incubated with trypomastigotes at $4^{\circ} \mathrm{C}$ or $37^{\circ} \mathrm{C}$. A concentration-dependent increase not only in the internal fluorescence but also in the percentage of fluorescent parasites was detected (Figures 1(h) and 1(i)). This confirms that EVs in the medium can be reincorporated.

3.2. EV Release Depends on Membrane Integrity. To test the hypothesis that EV release could depend on membrane integrity [17], parasites were pretreated with $\beta$-methyl- 


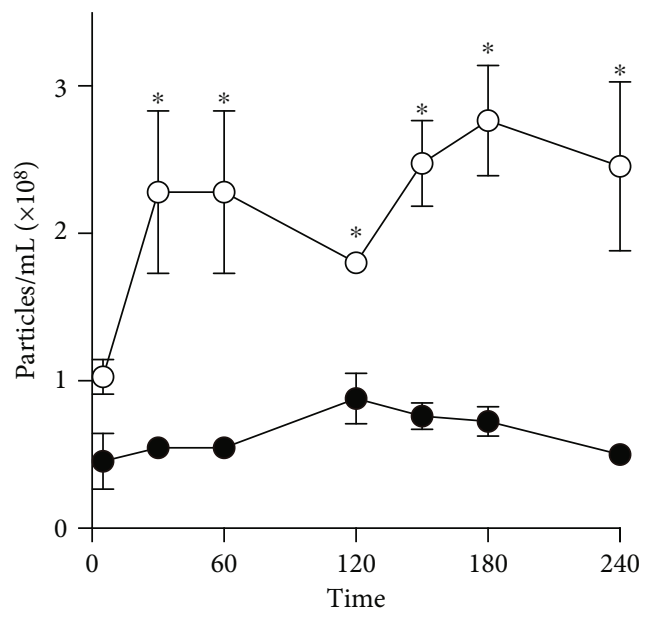

$-4^{\circ} \mathrm{C}$

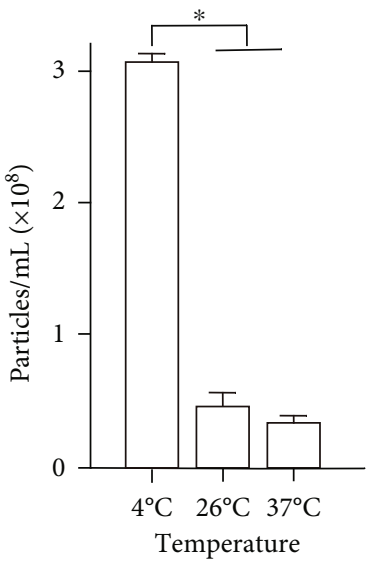

(b)

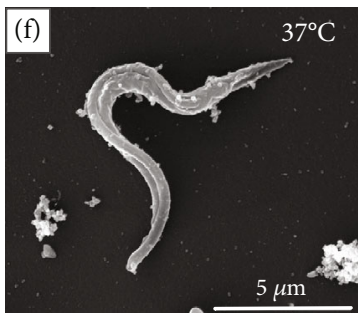

(f)

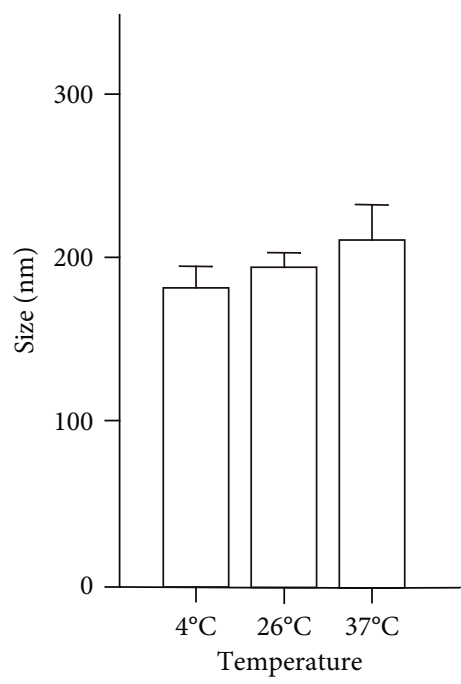

(c)

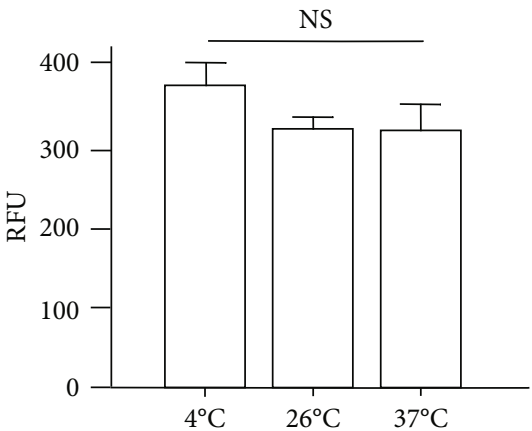

(g)

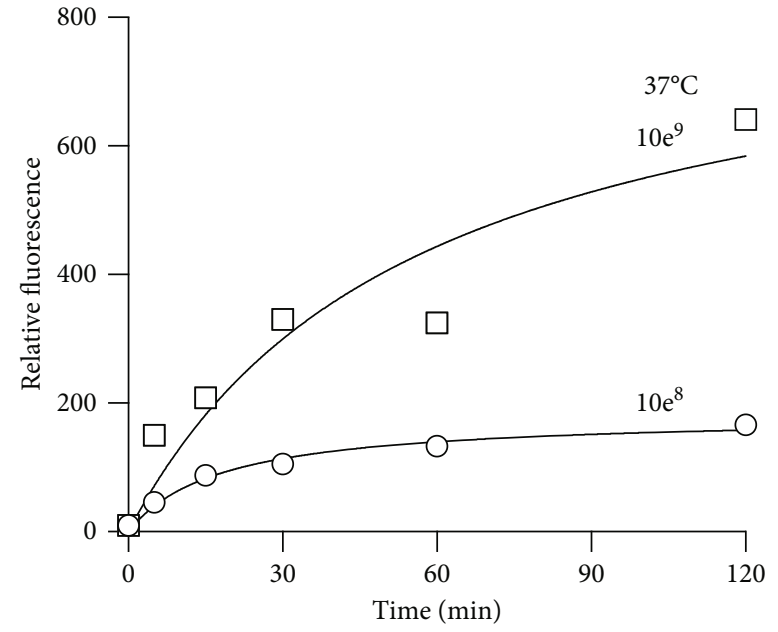

(h)

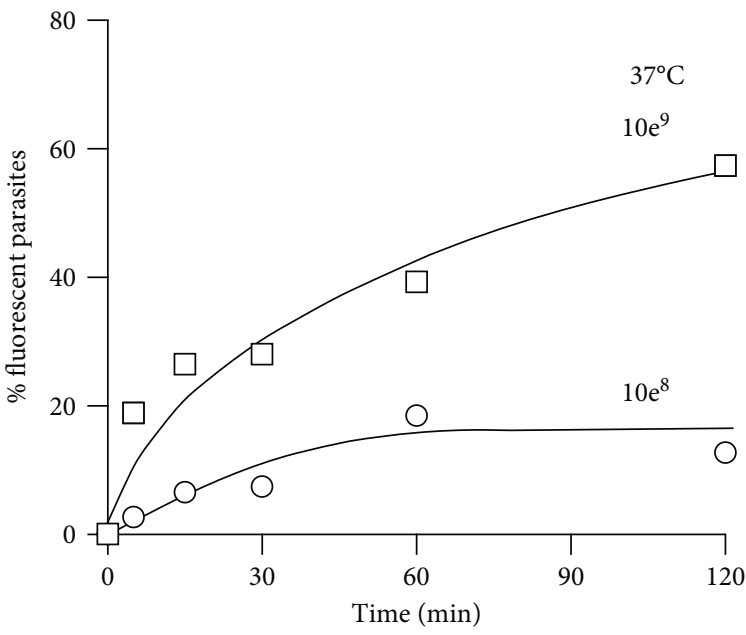

(i)

FIGURE 1: Kinetics of EV release by T. cruzi trypomastigote forms. $1 \times 10^{7}$ trypomastigotes were incubated in DMEM supplemented with $5 \%$ glucose at different temperatures. EV concentrations were determined at different time points by NTA (see Material and Methods). The panels show total EV concentration $(\mathrm{a}, \mathrm{b})$ and particle size (c) in the supernatants after $2 \mathrm{~h}$ of incubation at different temperatures $\left({ }^{*}<0.05\right)$. SEM images of trypomastigotes incubated for $2 \mathrm{~h}$ at $4^{\circ} \mathrm{C}(\mathrm{d}), 26^{\circ} \mathrm{C}(\mathrm{e})$, and $37^{\circ} \mathrm{C}((\mathrm{f})$, size bars $=5 \mu \mathrm{m})$. Panel $(\mathrm{g})$ indicates the correspondent relative fluorescence (RFU) of PrestoBlue viability reagent (mean and standard deviation, $n=3$ ) showing no significant (NS) differences $(p>0.05)$. EVs labeled with PKH26 $\left(1 \times 10^{8} / \mathrm{mL}\right.$ and $\left.10^{9} / \mathrm{mL}\right)$ were incubated with $1 \times 10^{7}$ trypomastigotes $/ \mathrm{mL}\left(37^{\circ} \mathrm{C}\right)$. The parasites were collected at different time points, and the median of fluorescence (h) or the percentage of labeled parasites (i) was evaluated by flow cytometry. Results are average values in triplicate measurements. 


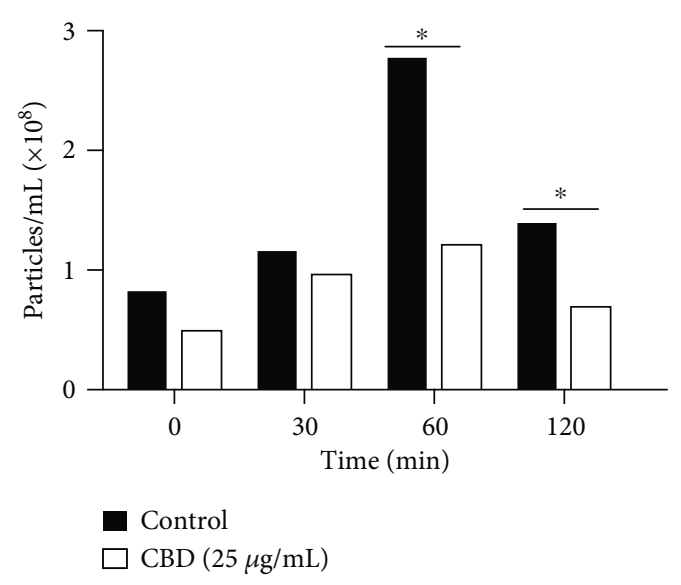

(a)

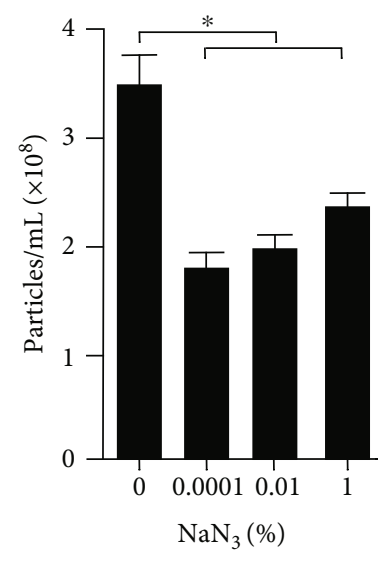

(b)

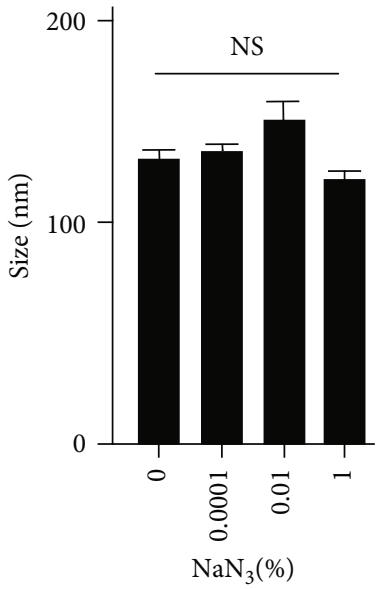

(c)
0

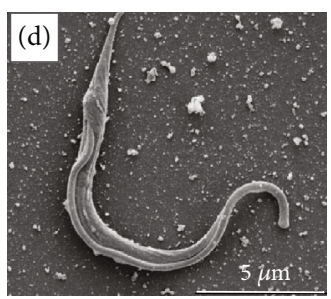

(d)

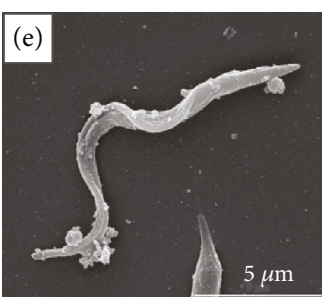

(e)

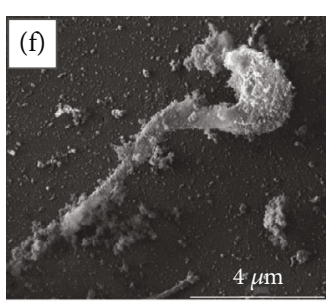

(f)

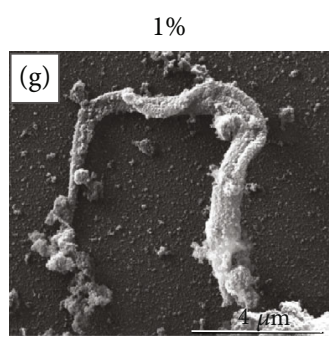

(g)

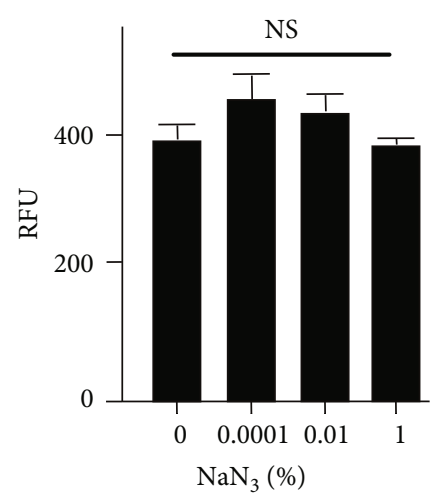

(h)

FiguRE 2: EV release (particles/mL), size $(\mathrm{nm})$, and membrane integrity under chemical stress. Trypanosoma cruzi trypomastigotes were incubated in the absence or presence of methyl-beta-cyclodextrin (CBD) $(25 \mu \mathrm{g} / \mathrm{mL})$ or $\mathrm{NaN}_{3}(0.0001$ to $1 \%)$ for $2 \mathrm{~h}$ at $37^{\circ} \mathrm{C}$. EVs in the supernatant were quantified by NTA in triplicate $\left({ }^{*} p<0.05\right)$. The panels show EV concentrations $(\mathrm{a}, \mathrm{b})$ and size $(\mathrm{nm})(\mathrm{c})$ in the presence of chemical agents. SEM of trypomastigotes preincubated with the indicated concentrations of $\mathrm{NaN}_{3}(\mathrm{~d}-\mathrm{g})$. Size bars are defined in each image. The relative fluorescence (RFU) of PrestoBlue viability reagent (mean \pm standard deviation, $n=3$ ) is shown in panel (h).

cyclodextrin (CBD), which affects trypomastigote structure by removing steroids from the membrane surface [18]. At lower concentrations of CBD $(0.02 \mathrm{mM})$, parasites released less EVs than controls (Figure 2(a)). Furthermore, the inhibition was not reestablished up to two hours after treatment, when no morphological changes were detected in the parasites. This suggests that small membrane perturbations affect EV secretion.

3.3. EV Discharge from the Parasite Is Dependent on Energy Sources. Since EV release increased at low temperature, the effect of sodium azide, known to partially inhibit T. cruzi oxidative phosphorylation and interaction $[19,20]$, was evaluated. The reductive capacity towards resazurin (PrestoBlue) was detected only at $1 \%$ of $\mathrm{NaN}_{3}$ (Figures $2(\mathrm{~g})$ and $2(\mathrm{~h})$ ). This suggests that even in the presence of azide, T. cruzi is still able to perform oxidative phosphorylation through a modified electron transport chain [19]. Sodium azide decreased the number of EVs in the supernatant of the parasite in all concentrations (Figure 2(b)), but no changes in the size was noticed. SEM images, however, showed that the parasite membrane became more granulated at higher 


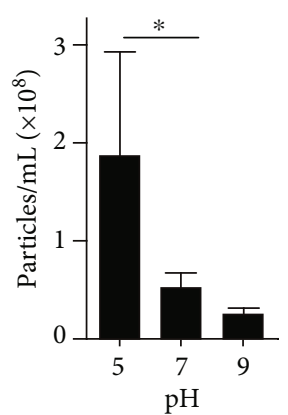

(a)

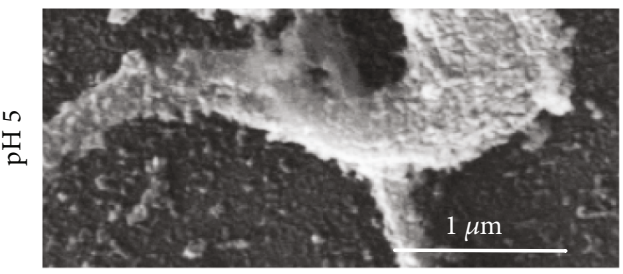

(c)

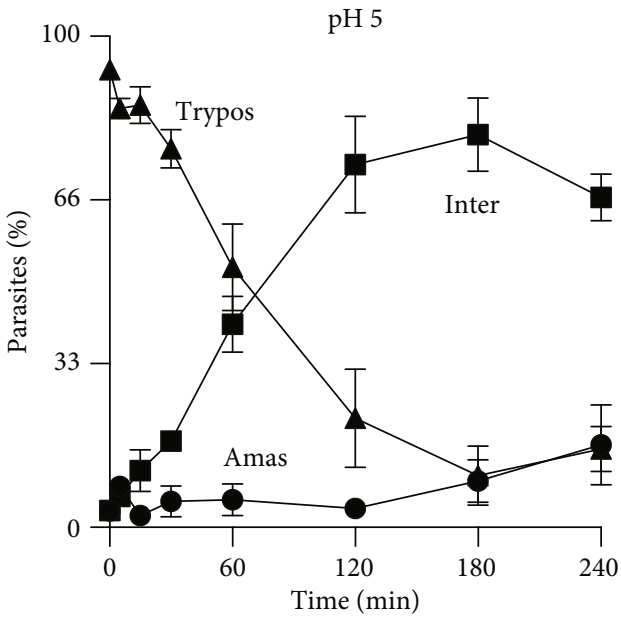

(e)

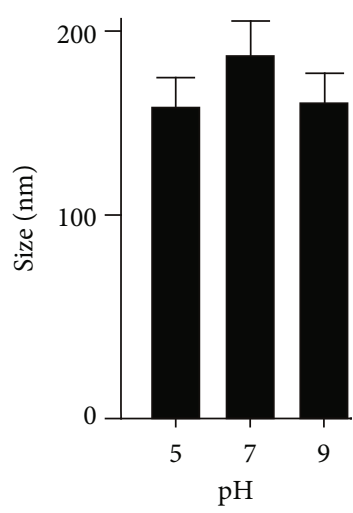

(b)

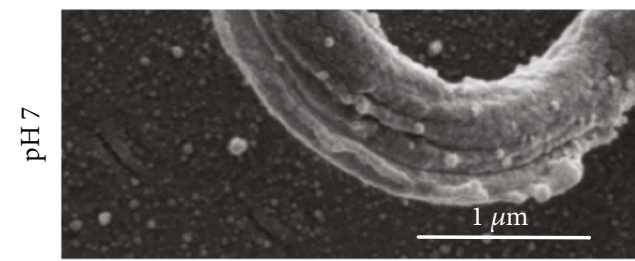

(d)

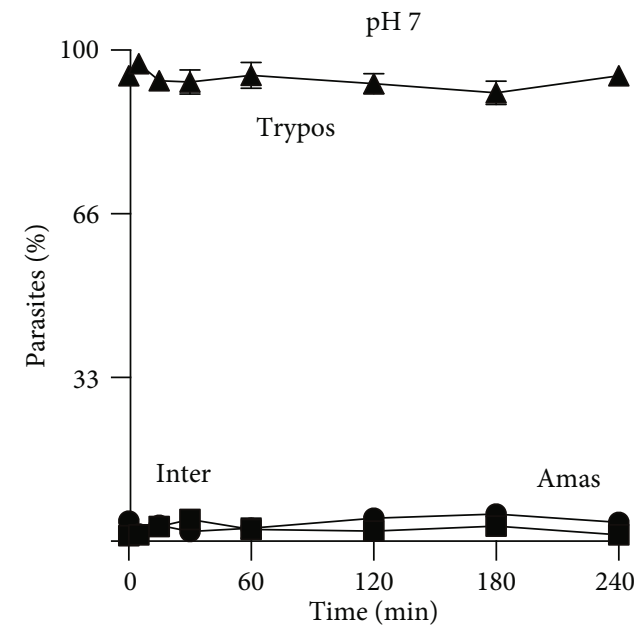

(f)

Figure 3: $\mathrm{pH}$ effect on EV release. Trypomastigote $\left(1 \times 10^{7} / \mathrm{mL}\right)$ forms incubated $\left(2 \mathrm{~h}, 37^{\circ} \mathrm{C}\right)$ in culture medium containing $5 \%$ glucose previously adjusted to the indicated $\mathrm{pH}$ values. EV concentrations (a) and sizes (b) were analyzed by NTA $\left({ }^{*} p<0.05\right)$. After incubation at pH 5 (c) or pH 7 (d), parasites were analyzed by SEM. In parallel, parasites were incubated at pH 5 (e) or pH 7 (f), stained with Giemsa, and 300 parasites were evaluated according to their form: trypomastigotes (Trypos), intermediate (Inter), or amastigote (Amas). Their percentages were quantified in triplicate.

$\mathrm{NaN}_{3}$ concentrations, and large blebs of parasite membrane are seen attached to the surface (Figures $2(\mathrm{~d})-2(\mathrm{~g})$ ).

3.4. Trypomastigote EV Release Is Boosted in Acidic Conditions. After mammalian cell invasion, the parasite faces $\mathrm{pH}$ changes. Therefore, we examined the release of EVs after $2 \mathrm{~h}$ of incubation at $\mathrm{pH} 5,7$, and 9. The parasite did not have significant changes in cell metabolic activity at $\mathrm{pH} 5$, a situation found inside cell lysosomes after cell invasion [21], while some decrease occurred at $\mathrm{pH}$ 9. The number of secreted EVs largely increased at pH 5 compared to $\mathrm{pH} 7$ and $\mathrm{pH} 9$ (Figure 3(a)), and there was no variation in the size of EVs (Figure 3(b)). This increased release can be seen by SEM images when trypomastigotes incubated under acidic conditions had more particles covering their surface compared to neutral conditions (Figures 3(c) and 3(d)). As already reported [22], at pH 5, trypomastigotes change shape and after 2 hours, they start to differentiate into amastigotes (Figure 3(e)). At pH 7, they remain unchanged (Figure 3(f)).

3.5. Effect of Nitrosative Stress in the EV Release. The major defense that eliminates $T$. cruzi in the mammalian host is nitrosative stress caused by nitric oxide (NO) [23]. We evaluated the effect of distinct $\mathrm{NaNO}_{2}$ concentrations, from 0 to $100 \mu \mathrm{M}$, at pH 5.0 on EV production. At this $\mathrm{pH}, \mathrm{NaNO}_{2}$ is soluble and spontaneously produces NO [24-27]. We 
observed a small effect in cell viability only at the concentration of $0.05 \mu \mathrm{M}$ (Figure 4(a)). However, a major decrease in the size of EVs (Figure 4(b)) along with an increase in $\mathrm{NaNO}_{2}$ was noticed (Figure 4(c)). SEM images showed that parasite integrity was conserved in all groups (Figure 4(d)).

The EV release induced by $\mathrm{NO}$ was confirmed by using S-nitrosoglutathione (SNOG), a NO generator that can be used at neutral $\mathrm{pH}$. Trypomastigotes were incubated in DMEM with SNOG $(100 \mu \mathrm{M})$, a toxic condition for cells. A decreased metabolic activity was detected (Figure 4(e)). In parallel, parasites released more EVs, reaching a maximum at 180 minutes without significant changes in size (Figures 4(f) and 4(g)) or morphology (Figure 4(h)). These results indicated that $\mathrm{EV}$ release by trypomastigotes is increased under nitrosative stress.

3.6. Immunomodulatory Role of EVs Released under Different Stress Conditions in BMDM. To investigate the immunomodulatory functions of EVs isolated under different stress conditions, IFN- $\gamma$-treated BMDM were exposed to them; differential gene expression was determined. In general, EVs activated BMDM in a similar manner of LPS, except for IL-12-p40 (Figure 5). No major differences were observed among the different types of EVs, suggesting that their functional cargo properties had a negligible effect under stress conditions.

\section{Discussion}

In nature, parasites deal with numerous environmental changes in vertebrate and invertebrate hosts, including variations in temperature, $\mathrm{pH}$, and reactive oxygen and nitrogen species. In fact, these factors modulate signaling cascades that regulate parasite infection, proliferation, and survival [28]. Previous studies have already shown that different stages of T. cruzi release EVs under physiological conditions. They modulate NO and cytokine production by macrophages and delay parasite migration in the gut of vector $[29,30]$. Here, several experiments evaluated the role of stress factors affecting EV release by $T$. cruzi trypomastigotes.

Studies on how temperature affects EV release in pathogens are very scarce. Early studies have already shown for Leishmania mexicana promastigotes, another trypanosomatid, that EV release increased at $37^{\circ} \mathrm{C}$, but not at $4^{\circ} \mathrm{C}$ [31]. Interestingly, for $T$. cruzi trypomastigotes, we found that larger amounts of EVs accumulate at low temperature $\left(4^{\circ} \mathrm{C}\right)$. Changes on the parasite surface organization, known to form patches containing different sets of components in the membrane, may be involved in this process in response to different environments [17]. Such distribution may change with temperature variations. For example, Leishmania promastigotes occur in the vector, where temperatures are around $25-26^{\circ} \mathrm{C}$, whereas $T$. cruzi trypomastigotes are mainly found in the mammalian host $\left(37^{\circ} \mathrm{C}\right)$. Most of the trypomastigote surface is composed of glycosylphosphatidylinositol- (GPI-) anchored proteins [32], and the lipid moieties are largely affected by decreasing the temperature. In fact, changes in temperature are largely sensed by $T$. cruzi triggering changes in gene expres- sion [33]. It is also possible that the accumulation was due to diminished reincorporation at low temperatures. However, this possibility is unlikely as the difference in the release at $4^{\circ} \mathrm{C}$ was rapid, much faster than the kinetics of reincorporation at $37^{\circ} \mathrm{C}$. Consistent with these observations, we observed that trypomastigote EVs are rapidly released and reincorporated. This may explain why after longer incubation periods, the amount of EVs in the supernatant reaches a saturation point. Uptake could be related to interparasite signaling as observed for T. brucei $[34,35]$. It secretes EVs as nanotubes that are also released in knockouts of the Vps36. This protein is part of the endosomal sorting complexes required for transport (ESCRT) involved in exosome formation. In T. cruzi metacyclic trypomastigotes, a stage that corresponds to infective forms generated in the insect vector, EVs confer serum complement resistance in susceptible parasites [9], but in general, paracrine signaling is poorly related in T. cruzi. Therefore, more studies are required to evaluate whether EV reincorporation has a role for mammalian stages of $T$. cruzi. Since temperature affects EV secretion, our next step was to evaluate if chemical agents could also modulate this phenomenon in T. cruzi trypomastigotes.

Our results showed that methyl- $\beta$-cyclodextrin (CBD) inhibited EV secretion in a concentration that maintains parasite integrity. This supports the idea that the process depends on the membrane bilayer organization. CBD removes sterol, required for the assembly of lipid domains, from membranes [36]. These domains are observed in trypomastigotes, and the presence of lipid chains and cholesterol or ergosterol boundaries might be involved in EV release. In fact, two different EV populations enriched in mucins and members of TS family of glycoproteins are detected [18]. CBD prevents Ca2+-dependent release of EVs from platelets [37] and other cells [38]. Further experiments are required to evaluate whether divalent cations affect $\mathrm{EV}$ release and the dependency of the negative charges provided by parasite sialylation. Azide inhibits trypomastigote adhesion to mammalian cells, most likely by preventing lateral diffusion of membrane components [20]. Like CBD, sodium azide also decreased EV production, although no major changes in parasite's viability were detected. Interestingly, at higher concentrations, the parasite surface became more granular, covered with several larger vesicles that remained attached to the membrane. In the same manner, it inhibits EV release. In summary, CBD and azide affected EV release by trypomastigotes without a negligible effect in the size. At higher concentrations, visible effects were detected in the parasite surface morphology.

During their life cycle, trypomastigotes enter mammalian cells, differentiate into amastigotes in parasitophorous vacuole, and can be retained in the cell, which is consistent with both the data in the literature and our own [39]. This is also a common described phenomenon $[22,40]$ when parasites grow for longer periods in acidified culture medium. Interestingly, here, EV release was increased under acidic conditions and the surface became granular, suggesting significant alterations in membrane. However, like azide, no major changes in the size were detected. Consistent with the literature, at $\mathrm{pH} 5$, trypomastigotes start to differentiate 


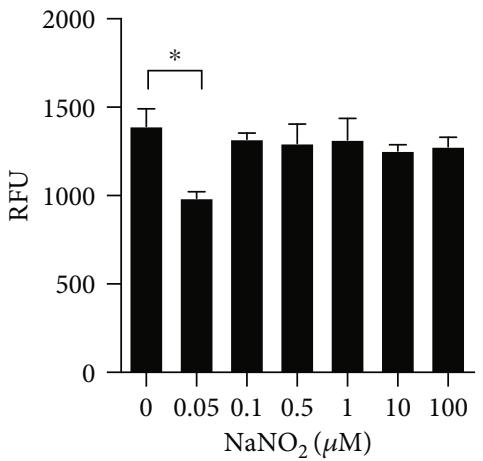

(a)

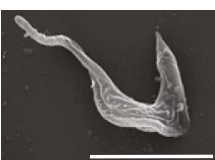

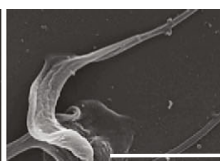

0.05

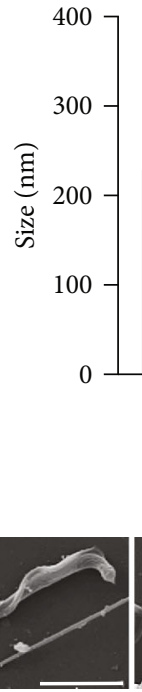

0.1

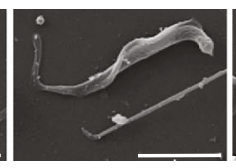

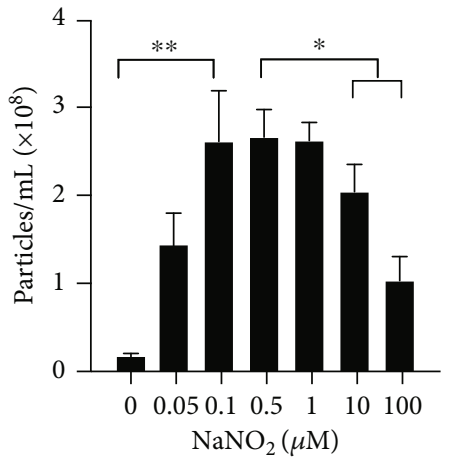

(c)

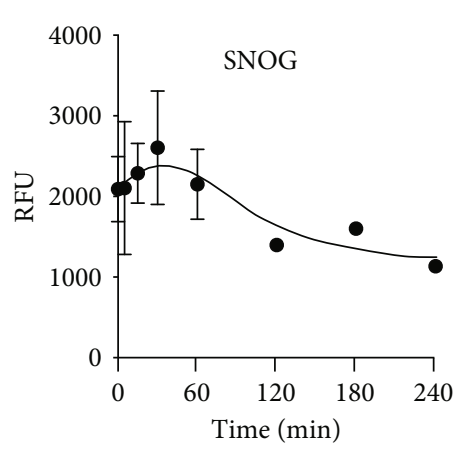

(e)

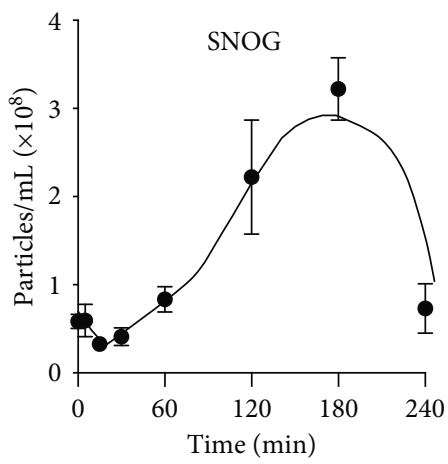

(g)

0

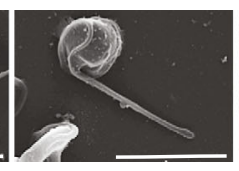

0.5
$\mathrm{NaNO}_{2}(\mu \mathrm{M})$

(d)

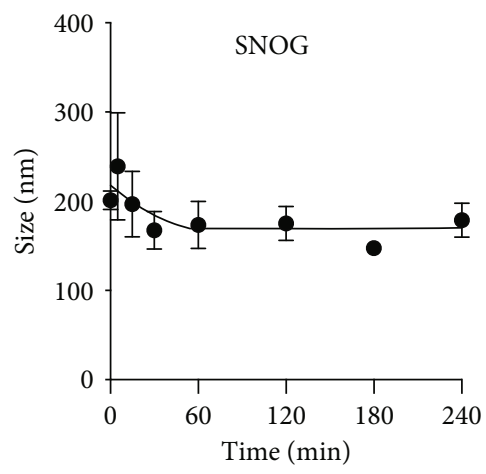

(f)

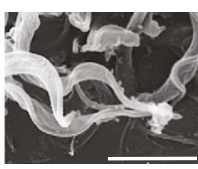

1

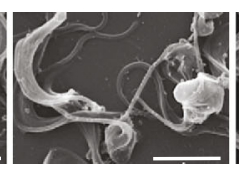

10

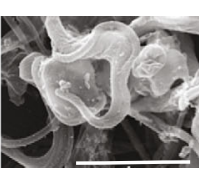

100
120

180

240

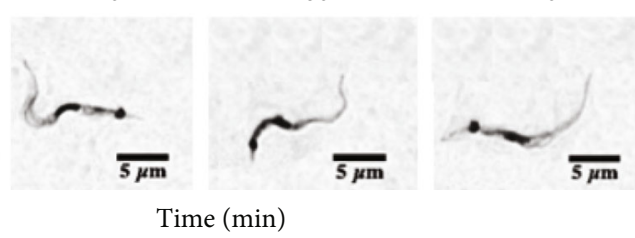

(h)

FIGURE 4: Effect of nitrosative stress on EV release by T. cruzi trypomastigotes. $1 \times 10^{7}$ trypomastigotes were incubated for $2 \mathrm{~h}$ at $37^{\circ} \mathrm{C}$ in culture medium adjusted to pH5.0 with different concentrations of $\mathrm{NaNO}_{2}$, including the control with the salt. After the incubation period, cell viability was evaluated by the PrestoBlue assay(a) and EV size (nm) (b) and EV concentration (particles/mL) (c) were measured by NTA $\left({ }^{*} p<0.05\right.$ and $\left.{ }^{* *} p<0.01\right)$. SEM of trypomastigotes releasing EVs incubated under different $\mathrm{NaNO}_{2}$ concentrations (d). Size bars $=5 \mu \mathrm{m}$. Alternatively, trypomastigotes were incubated at $37^{\circ} \mathrm{C}$ in DMEM supplemented with $5 \%$ glucose containing $100 \mu \mathrm{M}$ SNOG. Parasite viability was evaluated at the indicated intervals by PrestoBlue assays (e), and the size (f) and concentration (g) of EVs in the supernatants were determined by NTA $(n=3)$. Panel $(h)$ shows pictures of parasites stained by Giemsa after each incubation period.

into amastigote-like forms. Under acidic conditions, epimastigotes, the forms found in the vector, release membrane lipids [41]. Trypanosoma cruzi epimastigotes exhibit changes in morphology, especially in epimastigotes [42], which after $24 \mathrm{~h}$ induce large metabolic alterations [43]. However, it is still unclear how acidic conditions induce 


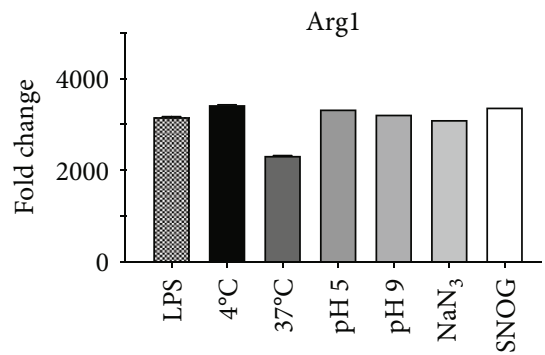

(a)

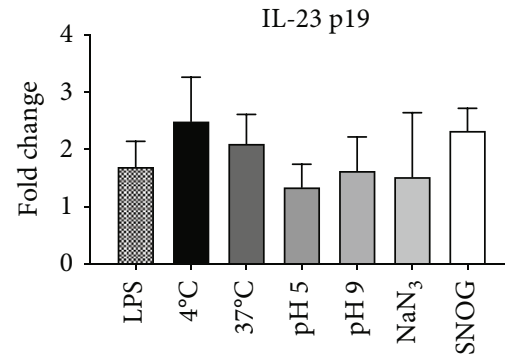

(c)

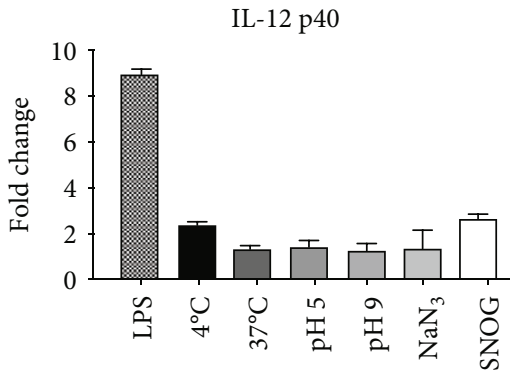

(e)

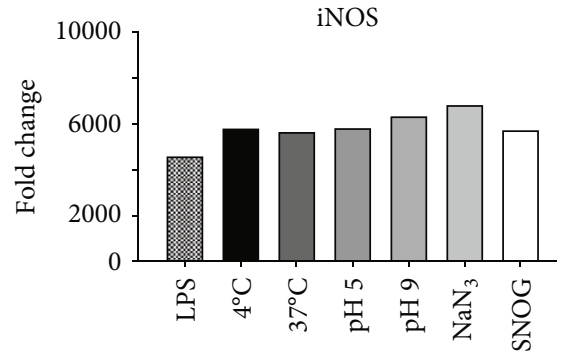

(b)

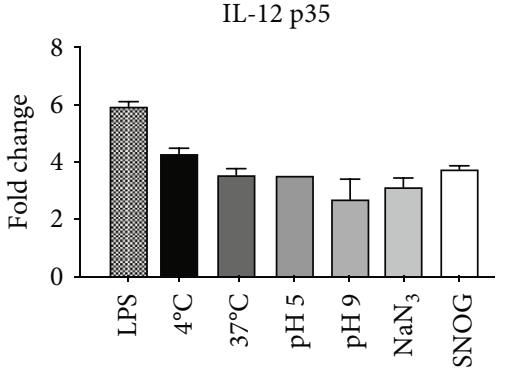

(d)

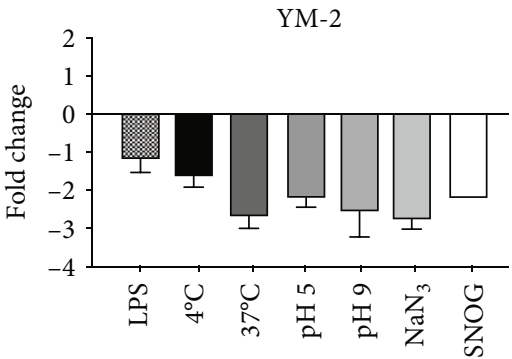

(f)

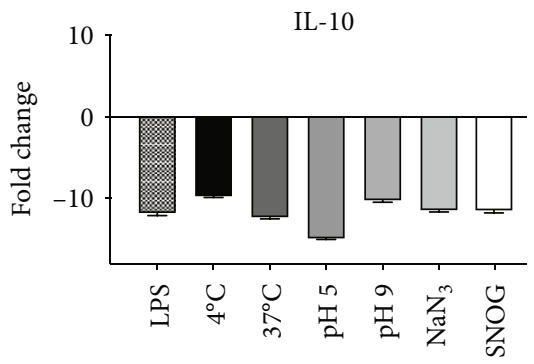

(g)

FIgURE 5: Immunomodulation of EVs obtained from T. cruzi submitted to different stress conditions. IFN- $\gamma$-primed macrophages (BMDM) were incubated $\left(24 \mathrm{~h}, 37^{\circ} \mathrm{C}\right)$ with $T$. cruzi EVs. LPS from E. coli was used as positive control. qRT-PCR was performed to detect the expression of immune response genes: Arg 1 (a), iNOS (b), IL-23 p19 (c), IL-12 p35 (d), IL-12 p40 (e), YM-2 (f), and IL-10 (g) ( $n=3$, $\left.{ }^{*} p<0.05\right)$.

parasite changes over short periods. The EV release may be increased by alterations in the subpellicular cytoskeleton interaction with the membrane at $\mathrm{pH} 5$ or through the activation of an acid-dependent and o-phenanthrolineinsensitive GPI-phospholipase present in trypomastigotes [44]. Interestingly, vesicles released by epimastigotes are enriched in phospholipids compared to the cell, but no increase in lysophospholipids was observed [45]. In a preliminary analysis, in trypomastigote EVs, we detected the presence of phosphatidylethanolamine and phosphatidylserine, absent in epimastigotes, by thin-layer chromatography and gas chromatography followed by MALDI spectrometry analysis (in preparation).

Finally, we decided to focus on nitrosative stress, since this is also a common environment faced by the parasites inside the cells. We also noticed that the release of EVs increased with NO-produced $\mathrm{NaNO}_{2}$ during medium acidification. A similar increase was observed at $\mathrm{pH} 7$ by using SNOG as a NO source. These effects are relevant, because the interaction of trypomastigotes and macrophages triggers the activation of the innate host immune response, characterized by the production of proinflammatory cytokines, 
activation of the NADPH oxidase complex, and the inducible nitric oxide synthase (iNOS) [46]. These enzymes produce radical superoxide $\left(\mathrm{O}_{2}^{-}\right)$and $\mathrm{NO}$, respectively [47]. These are responsible for the elimination of the parasite due to its trypanocide effect [48]. It is possible that the observed increase may be associated with the parasite's response to the stress suffered, a reflection of the mechanisms triggered by the protozoan to resist this stress, for example, via the trypanothione-dependent antioxidant system. Those events may be related to several factors, as a mechanism employed by the parasite to resist and survive nitrosative stress, involving intracellular signaling cascades that directly interfere with vesicular biogenesis. SNOG, as other NO donor molecules, is capable of inhibiting T. cruzi cruzipain (CZ), a cysteine protease, L. infantum cysteine proteinase, and $P$. falciparum falcipain by $S$-nitrosylation of the catalytic residue of the enzymes $[49,50]$. These observations might, therefore, suggest a role for this major surface protease in the trypomastigotes in EV release.

Trypanosoma cruzi EVs display high proinflammatory profile depending on the strain [51]. Consistent with these observations, here, the EVs increased the expression of iNOS, IL-12, and IL-23 genes, typical of M1-like phenotype, as previously observed [29]. EVs from T. cruzi contain a wide range of pathogen-associated molecular patterns (PAMPs) that contribute to their ability to interact with the cellular milieu in the vertebrate hosts. Those include GPI-mucins, trans-sialidases, and cruzipains [3]. Here, regardless of the stress conditions applied to the trypomastigotes, the functional properties of EVs were not affected. This suggests that stress conditions are more likely to affect quantity rather than quality of the EVs.

\section{Conclusion}

In conclusion, the release of $T$. cruzi EVs is dependent on factors found in the variable environmental conditions faced by the parasite. As the EVs confer increased infectivity and virulence in a mammalian host [2], the stress-induced release is clearly a response to environmental changes. We can associate the release with a mechanism of surface budding dependent on the membrane organization. This is based on the variable size of EVs, their composition like the plasma membrane, and the rapid release upon stress. It is still unknown whether the release occurs in a similar way in host tissues or in the blood and what key molecular events are taking place during the vesiculation and release from the parasite surface. This work provides an initial support to answer these relevant questions, to get a better understanding of Chagas disease and help to deal with it.

\section{Data Availability}

All data used to support the finding of this study are available from the corresponding authors upon request.

\section{Conflicts of Interest}

No potential conflicts of interest were reported by the authors.

\section{Authors' Contributions}

CIV, ACA, NS-M, JTM, WLB, PX, SS, and ACT conceived and designed the experiments. CIV, ACA, JTM, SS, WLB, and ACT performed most experiments. CIV, SS, RPS, and ACT wrote the manuscript. CIV, ACA, SS, PX, WLB, RPS, and ACT contributed to final manuscript revision.

\section{Acknowledgments}

This work was financially supported by Fundação de Amparo à Pesquisa do Estado de São Paulo (2015/20031-0, 2019/15909-0, and 2020/07870-4, to SS; 2017/02416-0); Fundação de Amparo à Pesquisa do Estado de Minas Gerais (PPM-00202-18); CNPq (445655/2014-3, 424729/2018-0, and $302972 / 2019-6$ and by the INCTVac-CNPq, to SS); and CAPES. We thank Claudio Rogerio for preparing the EVs, Nadjania Saraiva and Milena K Coló Brunialti for their help with flow cytometer analysis, and Lucas Barros for the help in some experiments.

\section{References}

[1] O World Health, Chagas Disease (Also Known as American Trypanosomiasis), 2021, https://www.who.int/news-room/ fact-sheets/detail/chagas-disease-(american-trypanosomiasis).

[2] A. C. Torrecilhas, R. P. Soares, S. Schenkman, C. FernandezPrada, and M. Olivier, "Extracellular vesicles in trypanosomatids: host cell communication," Frontiers in Cellular and Infection Microbiology, vol. 10, p. 602502, 2020.

[3] O. Campetella, C. A. Buscaglia, J. Mucci, and M. S. Leguizamon, "Parasite-host glycan interactions during Trypanosoma cruzi infection: trans -sialidase rides the show," Biochimica et Biophysica Acta. Molecular Basis of Diseases, vol. 1866, no. 5, p. 165692, 2020.

[4] A. TROCOLITORRECILHAS, R. TONELLI, W. PAVANELLI et al., "Trypanosoma cruzi: parasite shed vesicles increase heart parasitism and generate an intense inflammatory response," Microbes and Infection, vol. 11, no. 1, pp. 29-39, 2009.

[5] K. S. Ribeiro, C. I. Vasconcellos, R. P. Soares et al., "Proteomic analysis reveals different composition of extracellular vesicles released by two Trypanosoma cruzi strains associated with their distinct interaction with host cells," Journal of Extracellular Vesicles, vol. 7, no. 1, p. 1463779, 2018.

[6] L. Retana Moreira, A. Prescilla-Ledezma, A. Cornet-Gomez et al., "Biophysical and biochemical comparison of extracellular vesicles produced by infective and non-infective stages of Trypanosoma cruzi," International Journal of Molecular Sciences, vol. 22, no. 10, p. 5183, 2021.

[7] L. Retana Moreira, F. Rodriguez Serrano, and A. Osuna, "Extracellular vesicles of Trypanosoma cruzi tissue-culture cell-derived trypomastigotes: induction of physiological changes in non-parasitized culture cells," Plos Neglected and Tropical Diseases, vol. 13, no. 2, article e0007163, 2019.

[8] L. M. de Pablos Torro, L. Retana Moreira, and A. Osuna, "Extracellular vesicles in Chagas disease: a new passenger for an old disease," Frontiers in Microbiology, vol. 9, p. 1190, 2018.

[9] M. P. Wyllie and M. I. Ramirez, "Microvesicles released during the interaction between Trypanosoma cruzi TcI and TcII strains and host blood cells inhibit complement system and 
increase the infectivity of metacyclic forms of host cells in a strain-independent process," Pathogen and Disease, vol. 75, no. 7, 2017.

[10] I. V. Rossi, M. A. Ferreira Nunes, S. Vargas-Otalora, T. C. da Silva Ferreira, M. Cortez, and M. I. Ramirez, "Extracellular vesicles during TriTryps infection: complexity and future challenges," Molecular Immunology, vol. 132, pp. 172-183, 2021.

[11] C. Thery, K. W. Witwer, E. Aikawa et al., "Minimal information for studies of extracellular vesicles 2018 (MISEV2018): a position statement of the International Society for Extracellular Vesicles and update of the MISEV2014 guidelines," J Extracell Vesicles, vol. 7, no. 1, p. 1535750, 2018.

[12] D. S. Zamboni and M. Rabinovitch, "Nitric oxide partially controls Coxiella burnetii phase II infection in mouse primary macrophages," Infection and Immunity, vol. 71, no. 3, pp. 1225-1233, 2003.

[13] J. T. Maricato, M. N. Furtado, M. C. Takenaka et al., "Epigenetic modulations in activated cells early after HIV-1 infection and their possible functional consequences," PLoS One, vol. 10, no. 4, article e0119234, 2015.

[14] A. Cronemberger-Andrade, P. Xander, R. P. Soares et al., "Trypanosoma cruzi-infected human macrophages shed proinflammatory extracellular vesicles that enhance host-cell invasion via toll-like receptor 2," Frontiers in Cellular and Infection Microbiology, vol. 10, p. 99, 2020.

[15] T. D. Schmittgen and K. J. Livak, "Analyzing real-time PCR data by the comparative CT method," Nature Protocols, vol. 3, no. 6, pp. 1101-1108, 2008.

[16] S. A. Bustin, V. Benes, J. A. Garson et al., "The MIQE guidelines: minimum information for publication of quantitative real-time PCR experiments," Clinical Chemistry, vol. 55, no. 4, pp. 611-622, 2009.

[17] J. Mucci, A. B. Lantos, C. A. Buscaglia, M. S. Leguizamon, and O. Campetella, "The Trypanosoma cruzi surface, a nanoscale patchwork quilt," Trends in Parasitology, 2016.

[18] A. B. Lantos, G. Carlevaro, B. Araoz et al., "Sialic acid glycobiology unveils Trypanosoma cruzi trypomastigote membrane physiology," PLoS Pathogens, vol. 12, no. 4, article e1005559, 2016.

[19] A. O. M. Stoppani, R. Docampo, J. F. De Boiso, and A. C. C. Frasch, "Effect of inhibitors of electron transport and oxidative phosphorylation on Trypanosoma cruzi respiration and growth," Molecular and Biochemical Parasitology, vol. 2, no. 1, pp. 3-21, 1980.

[20] S. Schenkman, E. S. Robbins, and V. Nussenzweig, "Attachment of Trypanosoma cruzi to mammalian cells requires parasite energy, and invasion can be independent of the target cell cytoskeleton," Infection and Immunity, vol. 59, no. 2, pp. 645654, 1991.

[21] G. A. Mott and B. A. Burleigh, "The role of host cell lysosomes in Trypanosoma cruzi invasion," Sub-Cellular Biochemistry, vol. 47, pp. 165-173, 2008.

[22] S. Tomlinson, F. Vandekerckhove, U. Frevert, and V. Nussenzweig, "The induction of Trypanosoma cruzi trypomastigote to amastigote transformation by low $\mathrm{pH}$," Parasitology, vol. 110, no. 5, pp. 547-554, 1995.

[23] A. Santos-Miranda, J. V. Joviano-Santos, G. A. Ribeiro et al., "Reactive oxygen species and nitric oxide imbalances lead to in vivo and in vitro arrhythmogenic phenotype in acute phase of experimental Chagas disease," PLoS Pathogens, vol. 16, no. 3, article e1008379, 2020.
[24] J. Mauel, A. Ransijn, and Y. Buchmuller-Rouiller, "Killing of Leishmania parasites in activated murine macrophages is based on an L-arginine-dependent process that produces nitrogen derivatives," Journal of Leukocyte Biology, vol. 49, no. 1, pp. 73-82, 1991.

[25] T. Doi, M. Ando, T. Akaike, M. Suga, K. Sato, and H. Maeda, "Resistance to nitric oxide in Mycobacterium avium complex and its implication in pathogenesis," Infection and Immunity, vol. 61, no. 5, pp. 1980-1989, 1993.

[26] National Research Council (US), Committee on Nitrite, Alternative Curing Agents in Food, Assembly of Life Sciences (US), Committee on Nitrite, \& Alternative Curing Agents in Food, The Health Effects of Nitrate, Nitrite, and N-Nitroso Compounds: Part 1 of a 2-Part Study, The National Academies Press, Washington, DC, 1981.

[27] S. M. Brown, R. Upadhya, J. D. Shoemaker, and J. K. Lodge, "Isocitrate dehydrogenase is important for nitrosative stress resistance in Cryptococcus neoformans, but oxidative stress resistance is not dependent on glucose-6-phosphate dehydrogenase," Eukaryotic Cell, vol. 9, no. 6, pp. 971-980, 2010.

[28] K. Caradonna, J. Engel, D. Jacobi, C.-H. Lee, and B. Burleigh, "Host metabolism regulates intracellular growth of Trypanosoma cruzi," Cell Host \& Microbe, vol. 13, no. 1, pp. 108$117,2013$.

[29] P. M. Nogueira, K. Ribeiro, A. C. Silveira et al., "Vesicles from different Trypanosoma cruzi strains trigger differential innate and chronic immune responses," Journal of Extracellular Vesicles, vol. 4, no. 1, p. 28734, 2015.

[30] L. F. Paranaiba, A. A. Guarneri, A. C. Torrecilhas, M. N. Melo, and R. P. Soares, "Extracellular vesicles isolated from Trypanosoma cruzi affect early parasite migration in the gut of Rhodnius prolixus but not in Triatoma infestans," Memórias do Instituto Oswaldo Cruz, vol. 114, article ???, 2019.

[31] K. Hassani, E. Antoniak, A. Jardim, and M. Olivier, "Temperature-induced protein secretion by Leishmania mexicana modulates macrophage signalling and function," PLoS One, vol. 6, no. 5, article e18724, 2011.

[32] V. L. P. Chioccola, A. Acosta-Serrano, I. C. Almeida et al., "Mucin-like molecules form a negatively charged coat that protects Trypanosoma cruzi trypomastigotes from killing by human anti-alpha-galactosyl antibodies," Journal of Cell Science, vol. 113, no. 7, pp. 1299-1307, 2000.

[33] L. Cruz-Saavedra, M. Munoz, L. H. Patino, G. A. Vallejo, F. Guhl, and J. D. Ramirez, "Slight temperature changes cause rapid transcriptomic responses in Trypanosoma cruzi metacyclic trypomastigotes," Parasites and Vectors, vol. 13, no. 1, p. $255,2020$.

[34] D. Eliaz, S. Kannan, H. Shaked et al., "Exosome secretion affects social motility in Trypanosoma brucei," PLoS Pathogens, vol. 13, no. 3, article e1006245, 2017.

[35] A. J. Szempruch, L. Dennison, R. Kieft, J. M. Harrington, and S. L. Hajduk, "Sending a message: extracellular vesicles of pathogenic protozoan parasites," Nature Reviews. Microbiology, vol. 14, no. 11, pp. 669-675, 2016.

[36] R. Zidovetzki and I. Levitan, "Use of cyclodextrins to manipulate plasma membrane cholesterol content: evidence, misconceptions and control strategies," Biochimica et Biophysica Acta, vol. 1768, no. 6, pp. 1311-1324, 2007.

[37] H. Wei, J. M. Malcor, and M. T. Harper, "Lipid rafts are essential for release of phosphatidylserine-exposing extracellular vesicles from platelets," Scientific Reports, vol. 8, no. 1, p. 9987, 2018. 
[38] E. Sezgin, I. Levental, S. Mayor, and C. Eggeling, "The mystery of membrane organization: composition, regulation and roles of lipid rafts," Nature Reviews. Molecular Cell Biology, vol. 18, no. 6, pp. 361-374, 2017.

[39] L. O. Andrade and N. W. Andrews, "The Trypanosoma cruzi -host-cell interplay: location, invasion, retention," Nature Reviews. Microbiology, vol. 3, no. 10, pp. 819-823, 2005.

[40] H. Kanbara, H. Uemura, S. Nakasawa, and T. Fukama, "Effect of low $\mathrm{pH}$ on transformation of Trypanosoma cruzi trypomastigote to amastigote," Japanase Journal of Parasitology, vol. 39, pp. 226-228, 1990.

[41] J. F. da Silveira, P. A. Abrahamsohn, and W. Colli, "Plasma membrane vesicles isolated from epimastigote forms of trypanosoma cruzi," Biochimica et Biophysica Acta, vol. 550, no. 2, pp. 222-232, 1979.

[42] D. Perez-Morales, K. D. Hernandez, I. Martinez, L. T. Agredano-Moreno, L. F. Jimenez-Garcia, and B. Espinoza, "Ultrastructural and physiological changes induced by different stress conditions on the human parasite Trypanosoma cruzi," Cell Stress \& Chaperones, vol. 22, no. 1, pp. 15-27, 2017.

[43] Y. Pedra-Rezende, M. C. Fernandes, C. Mesquita-Rodrigues et al., "Starvation and $\mathrm{pH}$ stress conditions induced mitochondrial dysfunction, ROS production and autophagy in Trypanosoma cruzi epimastigotes," Biochimica et Biophysica Acta. Molecular Basis of Diseases, vol. 2021, no. 2, p. 166028, 2021.

[44] S. Schenkman, N. Yoshida, and M. L. Cardoso de Almeida, "Glycophosphatidylinositol-anchored proteins in metacyclic trypomastigotes of Trypanosoma cruzi," Molecular and Biochemical Parasitology, vol. 29, no. 2-3, pp. 141-151, 1988.

[45] J. F. Da Silveira and W. Colli, "Chemical composition of the plasma membrane from epimastigote forms of Trypanosoma cruzi," Biochimica et Biophysica Acta, vol. 644, no. 2, pp. 341-350, 1981.

[46] I. C. Almeida and R. T. Gazzinelli, "Proinflammatory activity of glycosylphosphatidylinositol anchors derived from Trypanosoma cruzi: structural and functional analyses," Journal of Leukocyte Biology, vol. 70, no. 4, pp. 467-477, 2001.

[47] L. Piacenza, M. N. Alvarez, G. Peluffo, and R. Radi, "Fighting the oxidative assault: the Trypanosoma cruzi journey to infection," Current opinion in microbiology., vol. 12, no. 4, pp. 415421, 2009.

[48] R. T. Gazzinelli, I. P. Oswald, S. Hieny, S. L. James, and A. Sher, "The microbicidal activity of interferon- $\gamma$-treated macrophages against Trypanosoma cruzi involves an L-argininedependent, nitrogen oxide-mediated mechanism inhibitable by interleukin-10 and transforming growth factor- $\beta$," European Journal of Immunology, vol. 22, no. 10, pp. 2501-2506, 1992.

[49] G. Venturini, L. Salvati, M. Muolo, M. Colasanti, L. Gradoni, and P. Ascenzi, "Nitric oxide inhibits cruzipain, the major papain-like cysteine proteinase from Trypanosoma cruzi," Biochemical and Biophysical Research Communications, vol. 270, no. 2, pp. 437-441, 2000.

[50] P. Ascenzi, A. Bocedi, M. Gentile, P. Visca, and L. Gradoni, "Inactivation of parasite cysteine proteinases by the NOdonor 4-(phenylsulfonyl)-3-((2-(dimethylamino)ethyl)thio)furoxan oxalate," Biochimica et Biophysica Acta, vol. 1703, no. 1, pp. 69-77, 2004.

[51] N. P. Nogueira, F. M. Saraiva, P. E. Sultano et al., "Proliferation and differentiation of Trypanosoma cruzi inside its vector have a new trigger: redox status," PLoS One, vol. 10, no. 2, article e0116712, 2015. 